\title{
GSP AND VCG BASED AUCTIONS FOR SPECTRUM SHARING IN COGNITIVE RADIO NETWORKS
}

\author{
SK.Surajjaha ${ }^{1}$, K.Annapurna ${ }^{2}$, B.Seetha Ramanjaneyulu ${ }^{3}$ \\ ${ }^{l}$ Department of Electronics and Communications, VFSTR University, Guntur, India \\ ${ }^{2}$ Department of Electronics and Communications, VFSTR University, Guntur, India \\ ${ }^{3}$ Department of Electronics and Communications, VFSTR University, Guntur, India
}

\begin{abstract}
Cognitive radio technology is a new mechanism proposed to address the spectrum related problems like under utilization by allotted users and scarcity to accommodate new users. Efficient sharing among competitive secondary users is one of the important tasks of cognitive radio systems. Auction based sharing is an efficient approach to allocate vacant channels to secondary users. In this paper, a cognitive radio network consisting of a central entity called primary owner (PO), many licensed users and many cognitive radio users (SUS) is considered. Each $S U$ bids for a channel to have the spectrum from the PO which acts as auctioneer. Generalized Second Price (GSP) and Vickrey-Clarke Grooves (VCG) auctions are implemented for sharing. Performance is measured in terms of Social welfare, Revenue of PO and SU utilities.
\end{abstract}

Keywords-Cognitive radio, Spectrum Sharing, Generalized Second Price Auction, Vickrey - Clarke Grooves auction $* * *$

\section{INTRODUCTION}

Continuous increasing demand of bandwidth from wireless devices of new age, many new services are facing the problem of spectrum scarcity. On the other side most of the allocated spectrum is not fully utilized. Only about $30 \%$ is in proper use, as per the spectrum usage measurements by FCC's spectrum policy task force [1]. This scenario underscores the importance of efficient utilization of wireless spectrum.

Cognitive Radio Technology is the best answer to address the issues of spectrum shortage and inefficient utilization of spectrum. It is based on intelligent radio concept which can automatically detects the available free channels and utilize them. Functioning of cognitive radio is illustrated in Figure1 , in which different functions of cognitive radio are shown [2]. The cognitive radio scans the spectrum and finds the un untilized spectrum bands i.e unoccupied channels at that time, at the given geographic location. These are also called as spectrum white spaces. The characteristics of the spectrum holes are analyzed. Depending on the user requirement of transmission, appropriate spectrum hole is selected for transmission. This whole phenomenon used by the cognitive radio is termed as cognitive radio cycle.

There are two main properties of cognitive radios. They are as follows:

i. Cognitive capability: This is the ability of cognitive radio to find out the vacant channels instantaneously with respect to both time and place.

ii. Re-configurability: This is the ability to change its transmission and reception parameters as it needs to change its transmission and reception on whatever the channel available to it[3].

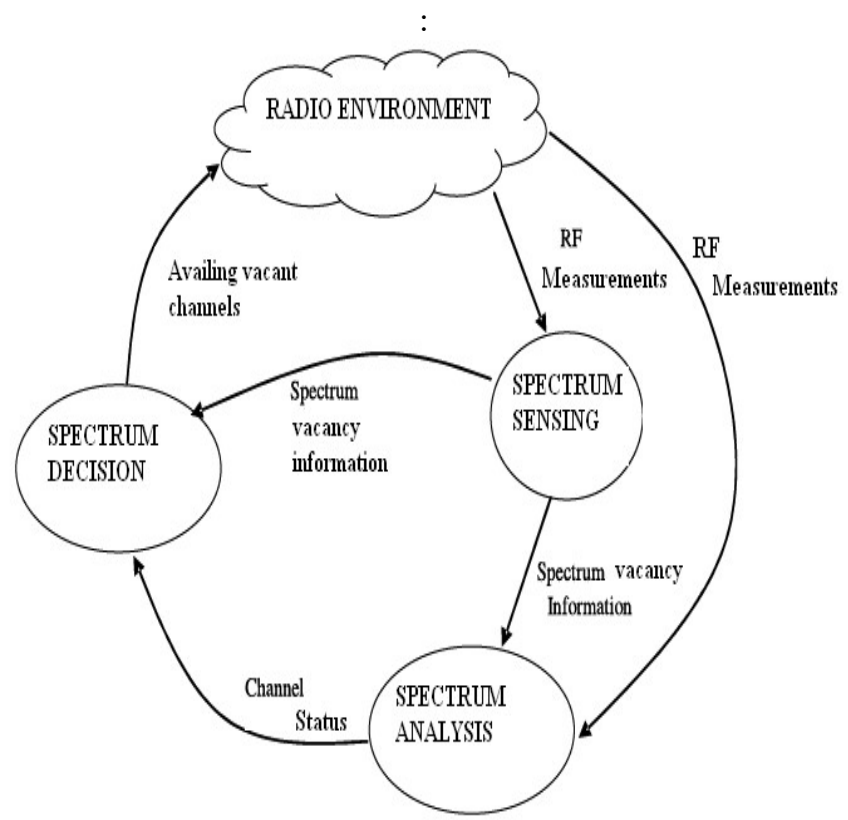

Fig-1: Cognitive Radio Life Cycle

\section{SPECTRUM SHARING TECHNIQUES}

There are various types of spectrum sharing techniques that are used in cognitive radio networks.

Centralized Spectrum Sharing: Here there exists a central unit that will take care of allotting available spectrum slots to the requesting secondary users at that time [4].

Distributed Spectrum Sharing: This type of sharing is implemented when sufficient infrastructure to manage the spectrum resources is not possible. In this scheme each individual user will detect the spectrum holes for their own utilization [4]. 
Cooperative Spectrum Sharing: The sensing information of all secondary users is shared among them selves and take cooperative decisions, which will aim at benefiting all the secondary users. The centralized spectrums sharing techniques will come under cooperative spectrum sharing [4]. Major problem with this type of sharing is confidentiality. [5]

Non-cooperative Spectrum Sharing: Here the secondary users individually sense the spectrum in their location and utilize the vacancies but don't share with other users. It is very much similar to distributed spectrum sharing. The advantage of this approach is that control traffic on common control channels is minimized and the channel allocation behavior is simplified.

Underlay Spectrum Sharing: In this type of sharing, the secondary users do not wait for vacant channels. They use the spectrum in the presence of primary users, but with less power so that they won't disturb the transmissions of primary users. Ultra Wideband (UWB) technologies use this kind of spectrum sharing [6].

Overlay Spectrum Sharing: In this sharing type the secondary users wait for vacancy of primary user to utilize the spectrum .

\section{GSP AND VCG BASED SPECTRUM}

\section{AUCTION}

In this work, spectrum sharing based on most popular auction based sharing schemes of Generalized Second Price Auction (GSP) and Vickrey - Clarke Grooves(VCG) is considered. The results of the schemes are compared to decide the suitable one for the given scenario.

GSP (Generalized Second Price Auction): This auction method works as follows. Each secondary user places a bid. The highest bidder will get a chance to use the first better channel, the second highest bidder will get a chance to use the second better channel and so on. But the highest bidder needs to the price bid of the second highest bidder, the second highest bidder needs to pay the price bid of third highest bidder and so on. Here for each channel the competing secondary users are observed and the highest bided secondary user is allotted that channel at the price of second highest bid.

Comparison of Generalized Second Price Auction with the commonly used "pay your bid auction" is as given below. In "pay your bid auction" dynamic stability problem is common. For example, if person ' $A$ ' bids for Rs.100 and another person ' $\mathrm{B}$ ' bids for Rs.80.The next time person $\mathrm{A}$ wants to bid for Rs81 instead of Rs100 as again he only will get the opportunity and by paying Rs19 less compared to previous. Then person B wants to bid for Rs82 and so on. This will create cat-mouse dynamics and to avoid such situation generalized second price auction came into existence, where bidders don't have as much incentive to bid below their value, because they will only pay the second highest bid if they win. GSP is a successful method of auction used in Internet for ad-space allotments.

Vickrey - Clarke Grooves auction: In this method, each secondary user which is utilizing the channel has to pay for the externality he causes, that is secondary user ' $i$ ' pays the difference between social welfare of the others with and without his participation [7]. Suppose there are two channels and three secondary users. Each secondary user bids for each channel. Let the following matrix represent bids by secondary users for each channel. Each row corresponds to each secondary user and each column corresponds to each channel.

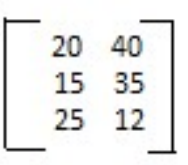

As per the bids placed, first channel will be assigned to user ' 3 ' and second channel will be assigned to user ' 1 '. According to VCG, the user ' 1 ' has to pay 'social welfare of PO from others without his presence' -'social welfare of PO from others with his presence' $=(35+25)-(25)=35$.

\section{SIMULATION RESULTS}

The amount obtained by PO with respect to number of secondary users for different number of channels is shown in Figure-2. As the number of channels increases, more opportunities will be availed by secondary users and more payment will be obtained by PO.

Social welfare of PO with respect to number of secondary users is shown in figure-3. Social welfare of PO is nothing but the amount to be got by PO as per the bids of secondary users, but not according to GSP. From Figure-3 it can be observed that social welfare is increasing with increase in number of secondary users and number of channels.

As mentioned earlier, increased number of channels increases the opportunities and increased number of secondary users increases the competition and hence social welfare.

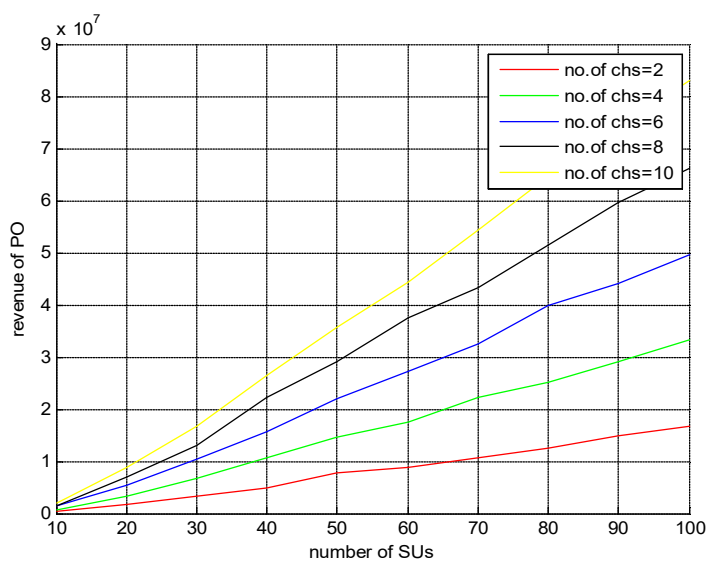

Fig-2: PO Revenue vs no.of secondary users in GSP 


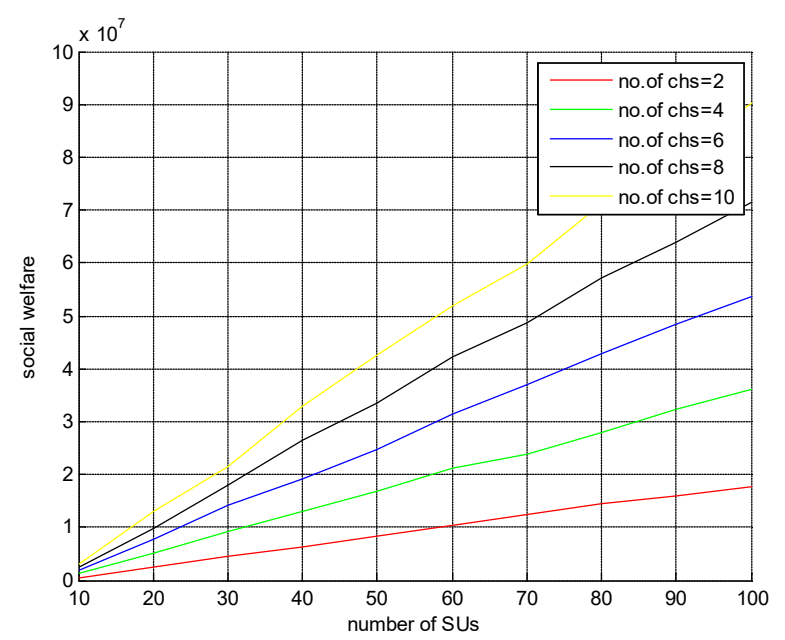

Fig-3: Social welfare of PO in GSP

Utility of secondary users is shown in figure-4. The difference between secondary user's actual bid values and paid values according to GSP is referred to as secondary user's utility. It is observed from figure-4 that when number of secondary users is increasing utilities of secondary user's increases initially and gets saturated afterwards.

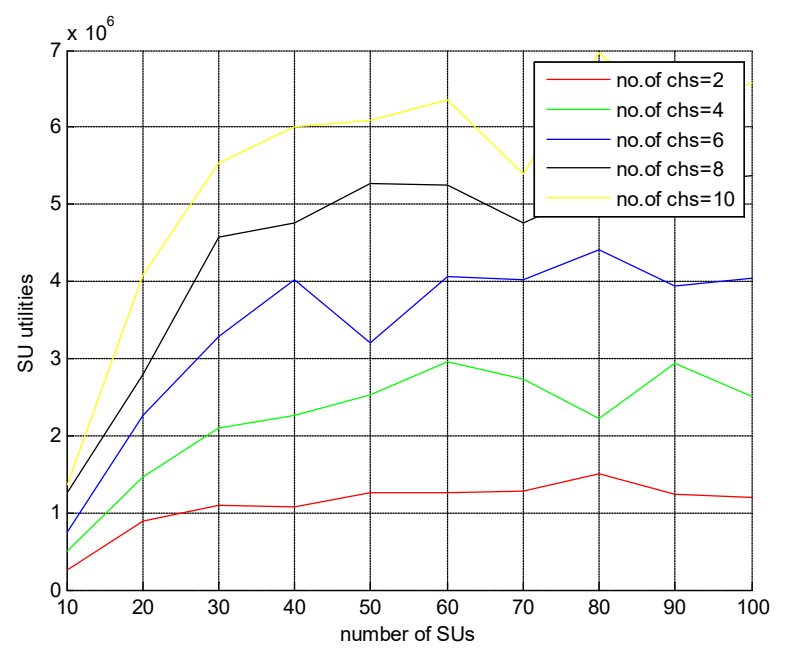

Fig-4: Secondary user utilities in GSP

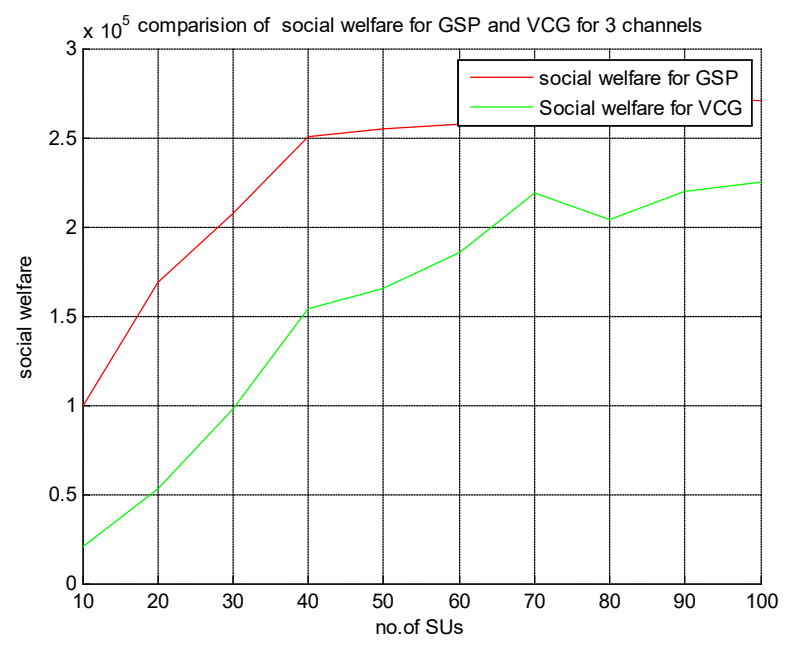

Fig-5: Comparison of social welfare

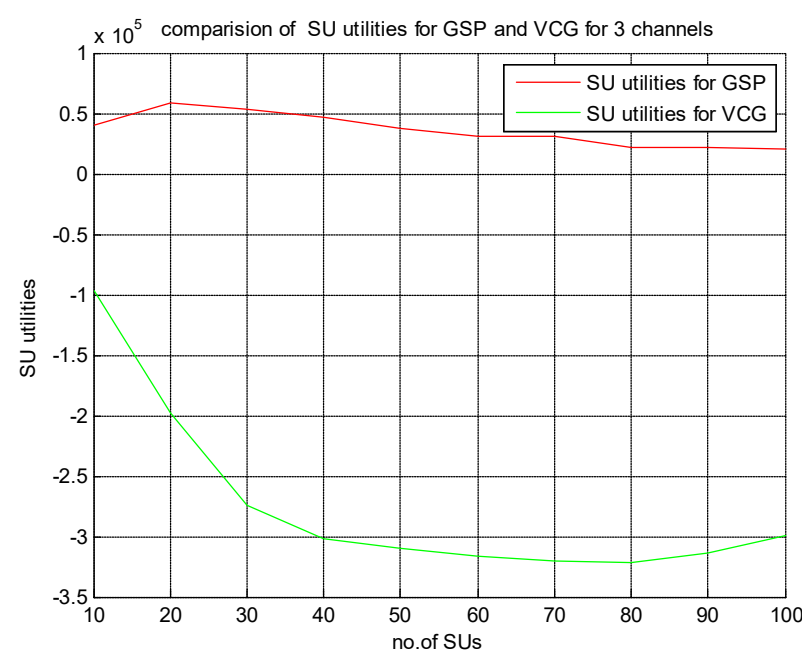

Fig-6: Comparison of SU utilities

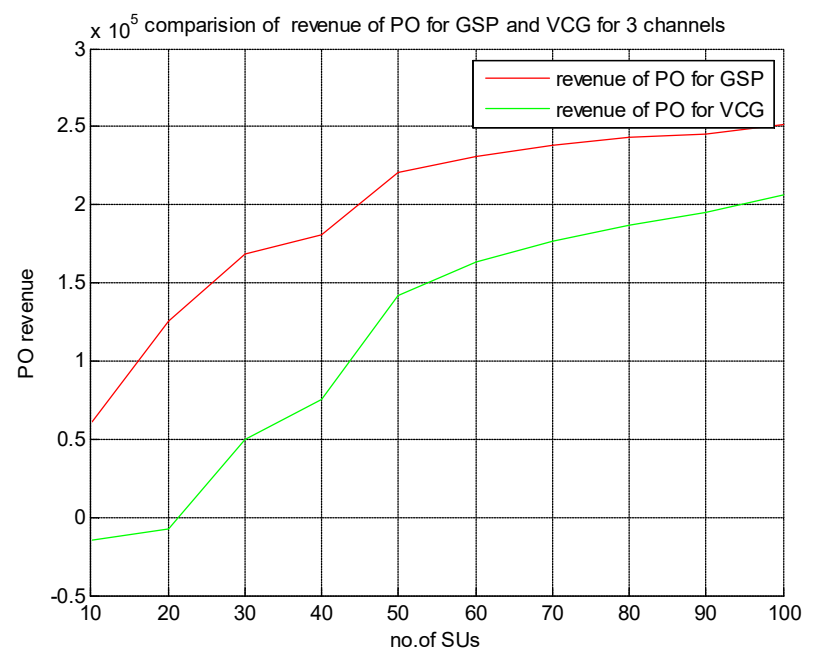

Fig-7: Comparison of PO revenue

From Figures 5,6 and 7, it can be observed that GSP is advantageous compared to VCG in both primary owner aspect and secondary users aspect as it gives more social welfare \& PO revenue compared to VCG's. It also offers better SU utilities.

\section{CONCLUSION}

In this paper we considered GSP and VCG auction methods for spectrum sharing in cognitive radio networks. In each case the secondary users bid for each channel. After bidding, price finalization and channel allotment will be done by checking the bids of each channel. By observing the results we can conclude that GSP is giving more profit to PO as well as secondary users.

\section{REFERENCES}

[1]. "Spectrum Policy Task Force report, Technical report 02-135", Federal Communications Commission, Nov. 2002. [2]. I.F.Akyildiz,W.Y. Lee and M.C.Vuran, "Next eneration/dynamic spectrum access/cognitive radio wireless networks: A survey," IEEE Transaction on Computer Networks, vol. 50, no. 13, pp. 2127-2159, May,2006. 
[3]. Daniel Willkomm, Robert Brodersen and Adam Wolisz, "A cognitive radio approach for usage of virtual unlicensed spectrum" in Proc. Of 14th IST Mobile Wireless Communications Summit, Dresden, Germany, 2005.

[4]. I.F. Akyildiz,W.Y.Lee and .C.Vuran "Next generation/dynamic spectrum access/cognitive radio wireless networks: A survey," IEEE Transaction on Computer Networks, vol. 50, no. 13, pp. 2127-2159, May,2006.

[5]. Honggang Zhang, Xiaofei Zhou and Tao Chen, "Cognitive Radio Networks", edited by Yang Xiao and Fei $\mathrm{Hu}, \mathrm{CRC}$ Press, 2008.

[6]. C. Ianculescu, A. Mudra., "Cognitive Radio and Dynamic Spectrum Sharing," in Proceeding of the SDR 05 Technical Conference and Product Exposition, 2005.

[7] Khaledi, Mehrdad, and Alhussein A. Abouzeid. "Auction-based spectrum sharing in cognitive radio networks with heterogeneous channels." Information Theory and Applications Workshop (ITA), 2013. IEEE, 2013. 